\title{
Legionella pneumophila Serogroup 2
}

National Cancer Institute

\section{Source}

National Cancer Institute. Legionella pneumophila Serogroup 2. NCI Thesaurus. Code C123499.

Subgroup 2 of Legionella pneumophila, differentiated by its antigenicity. Although this serogroup is rarely identified in infected individuals it causes clinical features similar to the pneumonia caused by other serogroups. 